\title{
The 12th International Conference on Model Transformations (ICMT 2019)
}

\author{
Regina Hebig ${ }^{\mathrm{a}} \quad$ Anthony Anjorin ${ }^{\mathrm{b}}$ \\ a. Chalmers | University of Gothenburg, Sweden \\ b. Paderborn University, Germany
}

The 12th International Conference on Model Transformations ICMT 2019 aims at advancing the state of knowledge about model transformation technologies. Model transformations are a core technology underlying the use of modeling and Domain Specific Languages (DLSs). Especially in DSL development, model transformations enable a decoupling of abstract and concrete syntax(es), supporting reuse and the coexistence of multiple concrete syntaxes (visual, textual) for the same DSL. Transformations also play a key role in analyzing models to reveal conceptual flaws or highlight quality bottlenecks, and in integrating heterogeneous tools into unified tool chains.

ICMT 2019 received 15 submissions and 6 papers were accepted, resulting in an acceptance rate of 40\%. ICMT 2019 includes an excellent keynote given by Paul Klint (CWI Research Fellow, University of Amsterdam, The Netherlands) on DSLs, the Good the Bad and the Ugly. ICMT 2019 will also feature a panel Is there a Future for Model Transformation Languages? that takes a critical look at the traditional approach(es) to designing model transformation languages and poses the question if these might become obsolete and be replaced with, e.g., approaches based on learning model transformations from examples.

The six accepted papers are:

1. Sebastian Ehmes, Lars Fritsche and Andy Schürr. SimSG: Rule-based Simulation using Stochastic Graph Transformation

2. Théo Le Calvar, Frédéric Jouault, Fabien Chhel and Mickael Clavreul. Efficient ATL Incremental Transformations

3. Eugene Syriani, Bill Robert and Manuel Wimmer. Domain-Specific Model Distance Measures

4. Heiko Klare, Torsten Syma, Erik Burger and Ralf Reussner. A Categorization of Interoperability Issues in Networks of Transformations

5. Nisha Desai and Martin Gogolla. Developing Comprehensive Postconditions Through a Model Transformation Chain

6. Stefan John, Alexandru Burdusel, Robert Bill, Daniel Strüber, Gabriele Taentzer, Steffen Zschaler and Manuel Wimmer. Searching for Optimal Models: Comparing Two Encoding Approaches 
Half of these papers focus on extensions and improvements of existing model transformation approaches including efficiency and incrementality (2), automating postcondition development in a transformation chain (5), and discussing issues that arise when handling networks of transformations (4). The other half of the papers explore novel application domains for model transformations such as stochastic simulations (1), and search-based optimisation (6) that requires good distance measures (3).

We would like to thank all the people who directly or indirectly contributed to ICMT 2019: the authors of all submissions, our reviewers, the participants of the conference, and the STAF 2019 organisers.

\author{
Anthony Anjorin \\ Regina Hebig \\ June 17, 2019
}

\title{
Organization
}

\section{Program Co-Chairs:}

Regina Hebig, Chalmers | University of Gothenburg, Sweden Anthony Anjorin, Paderborn University, Germany

\section{Program Committee:}

Alfonso Pierantonio, University of L'Aquila

Davide Di Ruscio, Università degli Studi dell'Aquila

Edward Willink, Willink Transformations Ltd.

Juan de Lara, Universidad Autónoma de Madrid, Spain

Yngve Lamo, Western Norway University of Applied Sciences

Gergely Varro, Independent

Jens Weber, University of Victoria

Reiko Heckel, University of Leicester

Marsha Chechik, University of Toronto

Esther Guerra, Universidad Autónoma de Madrid

Martin Gogolla, Database Systems Group, University of Bremen

Eugene Syriani, University of Montreal

Shekoufeh Kolahdouz Rahimi, University of Isfahan

Vadim Zaytsev, University of Amsterdam

Loli Burgueño, University of Málaga

Daniel Strüber, University of Koblenz and Landau

Dimitris Kolovos, University of York

Ludovico Iovino, Gran Sasso Science Institute, L'Aquila

Soichiro Hidaka, Hosei University

Malte Lochau, TU Darmstadt

Benoit Combemale, IRIT, University of Toulouse

Leen Lambers, Hasso-Plattner-Institut, Universität Potsdam

Manuel Wimmer, Business Informatics Group, Vienna University of Technology

Timo Kehrer, Humboldt-Universität zu Berlin

Massimo Tisi, IMT Atlantique, LS2N (UMR CNRS 6004)

Gabriele Taentzer, Philipps-Universität Marburg 